\title{
The effectiveness of tranexamic acid on operative and perioperative blood loss in long-segment spinal fusions: a consecutive series of 119 primary procedures
}

\author{
Mark N. Pernik, BA, ${ }^{1}$ Luke J. Dosselman, BS, ${ }^{1}$ Salah G. Aoun, MD, ${ }^{1}$ Adrienne D. Walker, BA, ${ }^{1}$ \\ Kristen Hall, BS, ${ }^{1}$ Valery Peinado Reyes, PA, ${ }^{1}$ David L. McDonagh, MD, ${ }^{2}$ and \\ Carlos A. Bagley, MD, MBA ${ }^{1}$
}

Departments of ${ }^{1}$ Neurological Surgery and ${ }^{2}$ Anesthesiology and Pain Management, UT Southwestern Medical Center Dallas, Texas

\begin{abstract}
OBJECTIVE The aim of this study was to determine if the use of tranexamic acid (TXA) in long-segment spinal fusion surgery can help reduce perioperative blood loss, transfusion requirements, and morbidity.

METHODS In this retrospective single-center study, the authors included 119 consecutive patients who underwent thoracolumbar fusion spanning at least 4 spinal levels from October 2016 to February 2019. Blood loss, transfusion requirements, perioperative morbidity, and adverse thrombotic events were compared between a cohort receiving intravenous TXA and a control group that did not.
\end{abstract}

RESULTS There was no significant difference in any measure of intraoperative blood loss (1514.3 vs $1209.1 \mathrm{~mL}, \mathrm{p}=$ 0.29 ) or transfusion requirement volume between the TXA and control groups despite a higher number of pelvic fusion procedures in the TXA group ( $85.9 \%$ vs $62.5 \%, p=0.003)$. Postoperative transfusion volume was significantly lower in TXA patients (954 vs $572 \mathrm{~mL}, p=0.01$ ). There was no difference in the incidence of thrombotic complications between the groups.

CONCLUSIONS TXA appears to provide a protective effect against blood loss in long-segment spine fusion surgery specifically when pelvic dissection and fixation is performed. TXA also seems to decrease postoperative transfusion requirements without increasing the risk of adverse thrombotic events.

https://thejns.org/doi/abs/10.3171/2019.11.SPINE191174

KEYWORDS tranexamic acid; long-segment fusion; operative transfusion; operative blood loss; spine surgery safety

\begin{abstract}
A $\mathrm{s}$ the population in the United States grows older, the number of surgical procedures for degenerative spine disease is bound to increase. ${ }^{1,23}$ Despite potential long-term quality of life benefits, reconstructive and multilevel spinal deformity surgery is not benign and can be complicated by significant operative morbidity, especially in older patients..$^{10,12}$ Due to the extent of surgical dissection, a feared but often anticipated complication is perioperative blood loss, which can be life-threatening if not addressed preemptively throughout the surgical case, and can be taxing on hospital resources, specifically the blood bank, given the need for significant transfusion volumes. $2,7,9,21,29,30$
\end{abstract}

To reduce the morbidity and need for poly-transfusions inherent to degenerative deformity spine surgery, several pharmacological approaches have been explored. ${ }^{30}$ The intraoperative use of tranexamic acid (TXA), an antifibrinolytic agent, has been shown to reduce the need for blood transfusion and intraoperative surgical blood loss across several specialties, including cardiac, orthopedic, and hepatic surgery. ${ }^{4,15}$ It is also used in most emergency department protocols as part of the management algorithm of polytrauma patients. ${ }^{26} \mathrm{TXA}$ is a lysine analog that inhibits the activation of plasminogen, decreasing the breakdown of fibrin thrombi to stabilize hemorrhage. TXA can be used as a topical, intravenous, or oral agent, but most in-

ABBREVIATIONS DVT = deep venous thrombosis; $E B L=$ estimated blood loss; $L O S=$ length of stay; PRBC = packed red blood cell; TXA = tranexamic acid. SUBMITTED September 26, 2019. ACCEPTED November 25, 2019.

INCLUDE WHEN CITING Published online January 24, 2020; DOI: 10.3171/2019.11.SPINE191174. 
vestigations have focused on its intravenous use. . $^{14,15,31,33-35}$ Because of its efficacy in reducing surgical bleeding in recent randomized controlled trials, it has gained increasing popularity in spine surgery. ${ }^{15,26,34,35}$ However, the administration of this agent is not benign, and has been associated with seizures and prothrombotic complications, specifically deep venous thrombosis (DVT) and pulmonary embolism, which are inherently more common after protracted spinal procedures. ${ }^{15}$

Currently, most studies on TXA in spine surgery either are limited by small sample sizes or involve cohorts with short posterior segment fusions. ${ }^{34,35}$ A less robust body of literature exists to support the efficacy and safety of TXA in complex spinal surgery, specifically long-segment deformity surgery that can involve pelvic dissection and fixation, where blood loss is high and multiple blood product transfusions are frequently necessary. $3,6,8,24,28,32$ While there are some data on the use of TXA in spinal deformity surgery suggesting that it might be safe, there are inconsistencies among studies regarding adequate dosage, the effect on perioperative blood loss and transfusion requirements, and the morbidity linked to its administration in larger procedures. ${ }^{3,6,18,24,28,32}$ Most studies on TXA in spinal deformity also include a significant proportion of revision procedures, which can be associated with additional blood loss due to longer operative time and a higher potential for operative complications. . $^{3,11,13,18,22,36}$

Our analysis aimed to investigate the impact of the intraoperative administration of TXA in first-time longsegment spinal fusion surgery, as it relates to blood loss reduction, intraoperative and postoperative transfusion needs, and thrombotic safety profile.

\section{Methods \\ Protocol}

The study protocol was approved by our institutional review board. This was a retrospective, single-center study that included 119 consecutive patients who underwent first-time spinal fusion of the thoracic or lumbar spine between October 2016 and February 2019 for degenerative spine disease. Patient data were prospectively collected in a spine registry and retrospectively reviewed using the electronic medical record system.

\section{Population Selection Criteria}

One hundred nineteen adults (age $\geq 18$ years) who had undergone thoracic or lumbar spinal fusion surgery for degenerative disease for the first time were retrospectively reviewed and included in the analysis. All patients had to have had 4 or more thoracic or lumbar segments fused. Exclusion criteria included patients who underwent cervical spine fusion exclusively, given the lower blood loss potential involved in these procedures, which obviates the need for TXA administration. Exclusion criteria also comprised patients who were undergoing a revision operation and patients who had infectious or metastatic spine disease.

The decision to administer TXA was done at the surgeon's discretion. The decision to give TXA was made preoperatively in all cases based on the surgeon's predic- tion of extent of dissection and length of procedure. Patients were divided into two groups: a treatment group that received intraoperative TXA (TXA group; $\mathrm{n}=71$ ) and a control group that did not (control; $\mathrm{n}=48$ ). TXA was given as a $10-\mathrm{mg} / \mathrm{kg}$ bolus followed by infusion of $1 \mathrm{mg} /$ $\mathrm{kg} / \mathrm{hr}$ per our anesthesia protocol. Cases were performed by 4 surgeons; however, $91.6 \%$ of cases were performed by a single surgeon who is the primary spinal deformity specialist in our group. All patients received standardized DVT prophylaxis including postoperative weight-based once-daily enoxaparin, compression socks during surgery and throughout their hospital stay, and physical therapyassisted walking on postoperative day 1 .

\section{Outcome Measures}

Outcome measures for this study included perioperative transfusions, operative and postoperative blood loss, ICU length of stay (LOS), hospital LOS, adverse thrombogenic events within 30 days, 90-day emergency department visits, and 30-day all-cause readmission. Given the variability in accuracy of estimated blood loss (EBL) and the unblinded nature of our study, we used additional objective intraoperative metrics of blood loss, including the percentage of hemoglobin decrease and the calculated blood loss. The difference between baseline hemoglobin (within 30 days of surgery) and postoperative hemoglobin (after completion of surgery) divided by the baseline value was calculated as the percentage of hemoglobin decrease. Calculated blood loss, as defined by Nadler and colleagues, was acquired using the percentage of hemoglobin decrease and the patient's calculated blood volume based on height, weight, and sex..$^{22,29}$ EBL was estimated as the difference between the total surgical suctioned volume and the total amount of irrigation used during the case. The hemoglobin concentration was measured in all patients preoperatively. All gauzes and lap sponges were wringed into the field and circulated through the suction device or cell saver and accounted for by EBL.

Perioperative transfusions were divided into those received intraoperatively and those received 48 hours postoperatively, as these are the periods where the most bleeding occurs in our experience. The 48-hour postoperative threshold is based on our experience with postoperative drain output. Drains tend to be pulled at the 48 -hour mark due to primarily serous output. We looked specifically at the summed intraoperative intravenous volume of autologous and allogeneic blood products administered, and the intraoperative packed red blood cell (PRBC) volume administered. Intraoperative transfusions were performed in accordance with the protocols of our anesthesiologists, who are experienced in deformity cases. An intraoperative hemoglobin target of $10 \mathrm{~g} / \mathrm{dL}$ is set. An increase in the requirements of phenylephrine to maintain normotension is often an indicator for verifying the hemoglobin concentration and initiating PRBC transfusion. Fresh frozen plasma is given after every $3 \mathrm{U}$ of PRBCs. Platelets are given after every $5 \mathrm{U}$ of PRBCs for a total concentration of $<10,0000 / \mu \mathrm{L}$. Cryoprecipitate is administered for a fibrinogen concentration of $<100 \mathrm{mg} / \mathrm{dL}$. Postoperative transfusions were compared in a similar fashion. The total intravenous volume of blood products was calculated 
using the added total volume of PRBCs, platelets, fresh frozen plasma, pooled cryoprecipitate, and/or cell salvage. The postoperative transfusion threshold for all patients undergoing spine surgery at our institution is below $9 \mathrm{~g} / \mathrm{dL}$ hemoglobin. All patients received complete blood counts on arrival to the ICU/postanesthesia care unit, daily for the first 3 postoperative days, and for any signs or symptoms of anemia.

Adverse events were defined as seizure, DVT (confirmed by ultrasound), pulmonary embolism (confirmed by CT angiography or high-probability ventilation-perfusion scan), ischemic myocardial infarction (confirmed by electrocardiography and laboratory work), or ischemic stroke (confirmed by CT or MRI) within 30 days of surgery. Adverse complications, readmissions, and emergency department visits both within and outside of our hospital system were captured through the medical record via follow-up notes, consults, and phone calls from all specialties for any indication of complications within 30 days or emergency department visits within 90 days.

Demographic and clinical variables were collected to assess potential differences between the TXA and control groups. Demographic variables included age, sex, BMI, height, and weight. Clinical variables included the number of levels of fusion, the presence of pelvic fixation, EBL, platelets, preoperative aspirin use, anesthesia time, and preoperative Cobb angle. Pelvic fixation was defined as the placement of iliac bolts. Follow-up for all patients was available for more than 16 months, although it is not relevant for the current study, and we typically follow longsegment fusion patients for longer than 5 years postoperatively at our center.

\section{Statistical Analysis}

We compared the outcome variables between the TXA and control group. Statistical analyses were performed using IBM SPSS (version 26, IBM Corp.). Descriptive statistics include mean and standard deviation (SD). For continuous variables we used a two-tailed t-test. The chi-square test was used to compare dichotomous variables between groups to assess differences in incidences. Statistical significance was set at $\alpha=0.05$.

\section{Results}

\section{Demographic and Imaging Characteristics}

Most patients were adult females (control group: 29 women and 19 men; TXA group: 49 women and 22 men). The average age was 69.2 years in the control group and 66.5 years in the TXA group. There were no differences between the groups when it came to age, sex, BMI, height, or weight (Table 1). The preoperative Cobb angle did not differ between the TXA and control groups.

\section{Clinical Characteristics}

A significantly higher proportion of patients in the TXA group underwent pelvic fixation than those in the control group $(85.9 \%$ vs $62.5 \%, \mathrm{p}<0.01)$. There was also a trend for TXA patients to undergo a greater number of spinal level fusions than the controls, although this variable did not reach statistical significance (9.18 vs 8.06, p
TABLE 1. Demographic data of the study population and the control group

\begin{tabular}{llll}
\hline \multicolumn{1}{c}{ Variable } & \multicolumn{1}{c}{ Control Group } & \multicolumn{1}{c}{ TXA Group } & p Value \\
\hline No. of patients & 48 & 71 & \\
\hline Mean age, $\mathrm{yrs}$ & $69.2(9.10)$ & $66.5(9.70)$ & 0.13 \\
\hline$\%$ male patients & $39.6 \%(\mathrm{n}=19)$ & $31.0 \%(\mathrm{n}=22)$ & $0.33^{*}$ \\
\hline Mean BMl, $\mathrm{kg} / \mathrm{m}^{2}$ & $28.6(8.04)$ & $30.3(7.10)$ & 0.23 \\
\hline Mean height, $\mathrm{m}$ & $1.70(0.09)$ & $1.70(0.11)$ & 0.40 \\
\hline Mean weight, $\mathrm{kg}$ & $82.3(23.5)$ & $85.2(19.9)$ & 0.48 \\
\hline Mean Cobb angle & $27.5^{\circ}(11.0) ; \mathrm{n}=21$ & $30.8^{\circ}(12.5) ; \mathrm{n}=48$ & 0.29 \\
\hline
\end{tabular}

Mean values are presented as mean (SD).

${ }^{*}$ Chi-square test.

$=0.061)$. Anesthetic duration was similar between TXA (305.9 minutes) and control (282.8 minutes) groups ( $\mathrm{p}=$ 0.22). Hemoglobin, calculated blood volume, aspirin use, and platelet count did not differ at the preoperative baseline between groups (Table 2).

\section{Perioperative Outcomes}

Major perioperative outcomes are summarized in Table 3. Intraoperative blood loss was not significantly different between the TXA and control groups when measured using EBL, mean hemoglobin decrease, or calculated blood loss. EBL was higher, but not in a statistically significant fashion, in the TXA group, largely due to high variance resulting from an outlier case (1838.4 vs $1494.7 \mathrm{~mL}, \mathrm{p}$ $=0.11$; see the asterisked footnote in Table 3). However, when EBL was controlled for the number of levels fused, the variance and the difference in magnitude normalized (EBL/level fused). Similarly, the volume of intraoperative transfusion of combined blood products (1514.3 vs 1209.1 $\mathrm{mL}, \mathrm{p}=0.29)$, the intraoperative volume of PRBCs administered (1042.9 vs $841.7 \mathrm{~mL}, \mathrm{p}=0.22)$, and the proportion of patients receiving an intraoperative transfusion (78.8\% vs $81.3 \%, \mathrm{p}=0.75)$ did not differ between the TXA and control groups.

In the first 48 hours postoperatively, TXA patients received significantly lower volumes of total blood products (954.2 vs 572.1 $\mathrm{mL}, \mathrm{p}=0.011)$ and PRBC volume (711.5 vs $478.9 \mathrm{~mL}, \mathrm{p}=0.026$ ) than those in the control group. Forty-two patients (59.2\%) in the control group and 32 patients $(66.7 \%)$ in the TXA group received transfusions at both timepoints $(p=0.41)$.

TXA patients had similar LOSs in the ICU (1.5 days) and hospital (5.7 days) compared with control patients (1.5 $[\mathrm{p}=0.91]$ and $5.8[\mathrm{p}=0.71]$ days, respectively). The percentage of patients in each group returning within 30 days for readmission or emergency department visits within 90 days was similar. The rate of thrombotic complications within 30 days after surgery was also not significantly different. Only one patient in the TXA group had a DVT that resolved with anticoagulation treatment. There were no seizures in either group.

\section{Discussion}

In our single-center retrospective study we aimed to 
TABLE 2. Clinical and operative data

\begin{tabular}{lccl}
\hline \multicolumn{1}{c}{ Variable } & Control Group & TXA Group & p Value \\
\hline Mean no. of levels fused & $8.1(2.78)$ & $9.2(3.41)$ & 0.061 \\
\hline \% pelvic fusion & $62.5 \%(n=30)$ & $85.9 \%(n=61)$ & $0.003^{*} \dagger$ \\
\hline Mean baseline $\mathrm{Hb}, \mathrm{g} / \mathrm{dL}$ & $13.4(1.69)$ & $13.4(1.26)$ & 0.98 \\
\hline $\begin{array}{l}\text { Mean calculated blood } \\
\text { vol, L }\end{array}$ & $5.1(0.90)$ & $5.1(0.84)$ & 0.76 \\
$\begin{array}{l}\text { Mean anesthesia dura- } \\
\text { tion, mins }\end{array}$ & $282.8(84.4)$ & $305.9(110.2)$ & 0.22 \\
\hline Mean baseline platelets & $235.2(68.1)$ & $241.4(59.2)$ & 0.60 \\
\hline$\%$ preop aspirin use & $37.5 \%(\mathrm{n}=18)$ & $28.2 \%(\mathrm{n}=20)$ & $0.28 \dagger$ \\
\hline
\end{tabular}

$\mathrm{Hb}=$ hemoglobin.

${ }^{*}$ Statistically significant $(p<0.05)$.

$\dagger$ Chi-square test.

investigate the effect of intraoperative intravenous TXA administration in cases of spinal fusion surgery spanning 4 or more thoracic or lumbar levels on surgical blood loss, blood product utilization, and morbidity including thromboembolic events. TXA has a favorable history of reducing blood loss in extensive surgical procedures ${ }^{15}$ however, its use in complex spinal deformity surgery is not as well studied. This is of importance to spine surgeons, since the effects of TXA on surgical blood loss may not be easily extrapolated to spinal surgery because of the thromboembolic risk inherent to the procedures. Deformity spine surgery is an independent risk factor for abnormal clot formation given the length of the procedure, the positioning of the patient, the extent of tissue dissection, and the mobilization of the major vessels with deformity correction. Based on previous literature, we postulated that TXA could reduce blood loss, transfusions, and morbidity after complex spine surgery. ${ }^{5}$ Our results appear to favor the use of TXA in extensive spine reconstructive procedures.

In our series, the TXA group underwent more extensive procedures with a significantly higher proportion of pelvic fusions and a trend toward a greater number of total vertebral levels fused (Tables 2 and 3). This difference largely stems from the nonrandomized nature of our study but may be due in part to bias of the surgeons who chose to administer TXA because of the predicted risk of significant hemorrhage in longer spinal constructs with greater extent of dissection. Previous studies on the inclusion of pelvic fixation in a spinal arthrodesis complex found that it independently increases blood loss, the need for perioperative transfusions, and postoperative morbidity. ${ }^{16,20,25,27}$ Considering these data, we should expect patients in the TXA group to lose more blood and require a greater amount of perioperative blood products than the control group since they have received more complex surgery. ${ }^{16,20,25,27}$ However, we observed no significant difference in any measure of intraoperative blood loss or utilization of blood products (Table 3). The similar amount of intraoperative blood loss in the TXA group despite larger procedures could be the result of a protective effect of TXA administration, since the two patient groups were otherwise similar at preoperative baseline.

Despite more extensive procedures in the TXA group, postoperative transfusion requirements were decreased. Patients receiving TXA received fewer units of total blood products and of PRBCs, indicating more efficient stabilization of postoperative hemorrhage compared with control (Table 3). The rates of transfusion in the postoperative period were relatively high in both groups $(>70 \%)$, but this was most likely due to our institution's relatively high transfusion threshold of $<9 \mathrm{~g} / \mathrm{dL}$ hemoglobin. In the case that our transfusion threshold was lower, the rates of transfusion may have been lower.

There are only a handful of retrospective series and small randomized controlled trials supporting the use of TXA in spine deformity. However, most of the studies are limited by their reliance on the surgeon's subjective operative EBL assessment to gauge efficacy, the inclusion of revision cases, small sample sizes, or variations in the administered intravenous dose of TXA. . $, 6,8,24,28,32$ Three retrospective series found that TXA reduced the necessity of blood transfusion perioperatively, and two of these studies reported reductions in EBL. 6,28,32 A randomized controlled trial conducted by Carabini and colleagues found that low-dose TXA reduced the intraoperative red blood cell transfusion volume by a median of $463 \mathrm{~mL}$ but had no effect on postoperative transfusions. ${ }^{3}$ This was not the case in our series, as the protective effect of TXA appeared to extend beyond the operating room, which is consistent with other retrospective series. ${ }^{6,28,32} \mathrm{~A}$ randomized trial by Peters and colleagues using doses similar to ours did not find any significant differences in measures of blood loss or transfusion. ${ }^{24}$ It is important to note that these trials are limited by their small sample sizes ( $\mathrm{n}<50$ patients), which may have prevented their analysis from detecting a significant clinical effect. ${ }^{3,8,24}$ Our results add to the existing TXA literature by analyzing intraoperative blood loss using objective and subjective measures and including only primary procedures to reduce heterogeneity in a patient sample that is relatively large.

We followed a low-dose TXA protocol in our series due to concerns for dose-dependent thrombotic adverse events and potential induction of seizures that have been reported in the cardiac literature. ${ }^{17,19}$ In available publications, the dose of intravenous TXA administered varied from 10 to $100 \mathrm{mg} / \mathrm{kg}$ for the loading dose followed by a 1- to $10-\mathrm{mg} / \mathrm{kg} / \mathrm{hr}$ infusion. ${ }^{3,6,8,24,28,32} \mathrm{Xie}$ and colleagues found that a reduction in surgical blood loss by as much as $50 \%$ required the highest dose of TXA $(100 \mathrm{mg} / \mathrm{kg}+$ $10-\mathrm{mg} / \mathrm{kg} / \mathrm{hr}$ infusion). ${ }^{32}$ The randomized trials, however, have all used relatively low-dose TXA protocols at $10 \mathrm{mg} /$ $\mathrm{kg}+1 \mathrm{mg} / \mathrm{kg} / \mathrm{hr} r^{3,6,8,24}$ or $20 \mathrm{mg} / \mathrm{kg}^{8}$ and had mixed results. Current literature on TXA use in spine surgery supports a dose-dependent effect on blood loss without increases in adverse events. ${ }^{5,18,34}$ Unfortunately, meta-analysis data are not specific to spine surgery, and final conclusions regarding the optimal administration dose cannot be made at this time. ${ }^{5,34}$ It is possible that higher TXA doses could have been more effective in reducing blood loss in our study without compromising safety. A safety series of 100 spinal deformity cases using $50 \mathrm{mg} / \mathrm{kg}$ and $5-\mathrm{mg} / \mathrm{kg} / \mathrm{hr}$ infusions found a $3 \%$ incidence of thrombotic events (1 pulmonary embolism and 2 DVTs) with no reported seizures, strokes, or myocardial infarctions, indicating that 
TABLE 3. Perioperative outcome measures

\begin{tabular}{|c|c|c|c|}
\hline Variable & Control Group & TXA Group & $p$ Value \\
\hline $\mathrm{EBL}, \mathrm{mL}$ & $1494.7(863.0)$ & $1838.4(1293.8)^{*}$ & 0.11 \\
\hline EBL/level fused & $181.5(51.9)$ & $187.7(43.8)$ & 0.48 \\
\hline Calculated blood loss, $\mathrm{mL}$ & $1322.7(671.8)$ & $1318.0(673.2)$ & 0.97 \\
\hline Calculated blood loss/level fused & $180.99(104.4)$ & $155.64(92.42)$ & 0.17 \\
\hline$\%$ receiving intraop transfusion & $81.3 \%(n=39)$ & $78.8 \%(n=56)$ & $0.75 \dagger$ \\
\hline Intraop blood products, $\mathrm{mL}$ & $1209.1(1281.7)$ & $1514.3(1668.3)^{*}$ & 0.29 \\
\hline Intraop PRBCs, mL & $841.7(717.2)$ & $1042.9(967.5)^{*}$ & 0.22 \\
\hline Postop platelets & 149.4 & 153.3 & 0.68 \\
\hline Postop Hb, g/dL & $9.8(1.6)$ & $9.8(1.5)$ & 0.92 \\
\hline Mean $\mathrm{Hb}$ drop, \% & $25.9 \%(12.1 \%)$ & $25.8 \%(12.9 \%)$ & 0.96 \\
\hline$\%$ receiving postop transfusion & $79.2 \%(n=38)$ & $74.6 \%(n=53)$ & $0.57 \dagger$ \\
\hline Total postop blood products, $\mathrm{mL}$ & $954.2(1043.1)$ & $572.1(572.0)$ & $0.011 \ddagger$ \\
\hline Postop PRBCs, mL & $711.5(669.6)$ & $478.9(457.6)$ & $0.026 \ddagger$ \\
\hline$\%$ receiving intra- $\&$ postop transfusions & $59.2 \%(n=42)$ & $66.7 \%(n=32)$ & $0.41 \dagger$ \\
\hline ICU LOS, days & $1.5(1.1)$ & $1.5(1.0)$ & 0.91 \\
\hline Total LOS, days & $5.8(1.8)$ & $5.7(1.7)$ & 0.71 \\
\hline$\%$ readmitted (w/in 30 days) & $10.4 \%(n=5)$ & $5.6 \%(n=4)$ & $0.33 \dagger$ \\
\hline \% ED visit (w/in 90 days) & $10.4 \%(n=5)$ & $9.9 \%(n=7)$ & $0.92 \dagger$ \\
\hline$\%$ thrombotic events (w/in 30 days) & $0.0 \%(n=0)$ & $1.4 \%(n=1)$ & $0.41 \dagger$ \\
\hline$\%$ seizures & $0.0 \%(n=0)$ & $0.0 \%(n=0)$ & \\
\hline
\end{tabular}

Values are mean (SD) unless otherwise indicated.

* One patient is included in the TXA group analysis despite intraoperative pulseless electrical activity and coagulopathy requiring chest compressions and massive blood transfusions. Intraoperative transfusion volume (8861 mL), EBL (6000 $\mathrm{mL})$, and PRBC volume (5400 mL) were all greater than 3 SD from the mean. We found no evidence that the acute hemodynamic instability was caused by TXA administration but included the data for completeness.

† Chi-square test.

$\ddagger$ Statistically significant $(p<0.05)$.

higher doses may be safe. ${ }^{18}$ High-dose regimens may be considered in patients with higher relative bleeding risks.

\section{Study Limitations}

Our study has several limitations. First, because of the retrospective nature of data review, it is at risk for recall and selection bias. In addition, the decision to administer TXA was left to the surgeon's discretion. Selection bias may be highlighted in our series since the TXA group included a greater proportion of pelvic fixation. Despite this bias, the two groups had statistically comparable demographic characteristics preoperatively, and the Cobb angle of the deformities was similar, which could imply that the cases represented similar surgical difficulty. Another limitation of our study is the inclusion of multiple surgeons, whose surgical technique and perioperative management may differ and affect perioperative transfusions. While our study measures thrombotic complications using a lowdose TXA protocol, the size of our study may not be large enough to draw definitive conclusions on the safety of TXA in complex spine surgery. A meta-analysis focused on the safety of TXA use in the complex spine is likely needed to address this question more robustly. Finally, both groups were heterogeneous in terms of the specific procedures, even if they fell within the same category of long-segment fusion of 4 or more vertebral levels.

\section{Conclusions}

TXA administration can be helpful in reducing intraoperative and postoperative blood loss and the need for blood product transfusion in patients undergoing complex thoracolumbar deformity surgery, especially when pelvic fixation is involved. There appears to be no added thromboembolic morbidity with low-dose protocols. In addition, the hemostatic effect of TXA appears to extend beyond the operative time and may reduce the need for transfusion in the 48 hours following surgery. A well-powered, multicenter randomized trial with homogeneous, carefully selected surgical procedures will be needed to properly assess the clinical effect of variations in dosages and the resulting adverse events.

\section{References}

1. Ames CP, Scheer JK, Lafage V, Smith JS, Bess S, Berven $\mathrm{SH}$, et al: Adult spinal deformity: epidemiology, health impact, evaluation, and management. Spine Deform 4:310322,2016

2. Bess RS, Lenke LG, Bridwell KH, Steger-May K, Hensley $\mathrm{M}$ : Wasting of preoperatively donated autologous blood in the surgical treatment of adolescent idiopathic scoliosis. Spine (Phila Pa 1976) 31:2375-2380, 2006

3. Carabini LM, Moreland NC, Vealey RJ, Bebawy JF, Koski TR, Koht A, et al: A randomized controlled trial of low- 
dose tranexamic acid versus placebo to reduce red blood cell transfusion during complex multilevel spine fusion surgery. World Neurosurg 110:e572-e579, 2018

4. Chen JY, Chin PL, Moo IH, Pang HN, Tay DK, Chia SL, et al: Intravenous versus intra-articular tranexamic acid in total knee arthroplasty: a double-blinded randomised controlled noninferiority trial. Knee 23:152-156, 2016

5. Cheriyan T, Maier SP II, Bianco K, Slobodyanyuk K, Rattenni RN, Lafage V, et al: Efficacy of tranexamic acid on surgical bleeding in spine surgery: a meta-analysis. Spine $\mathbf{J}$ 15:752-761, 2015

6. Choi HY, Hyun SJ, Kim KJ, Jahng TA, Kim HJ: Effectiveness and safety of tranexamic acid in spinal deformity surgery. J Korean Neurosurg Soc 60:75-81, 2017

7. Cole JW, Murray DJ, Snider RJ, Bassett GS, Bridwell KH, Lenke LG: Aprotinin reduces blood loss during spinal surgery in children. Spine (Phila Pa 1976) 28:2482-2485, 2003

8. Colomina MJ, Koo M, Basora M, Pizones J, Mora L, Bagó J: Intraoperative tranexamic acid use in major spine surgery in adults: a multicentre, randomized, placebo-controlled trial. Br J Anaesth 118:380-390, 2017

9. Copley LA, Richards BS, Safavi FZ, Newton PO: Hemodilution as a method to reduce transfusion requirements in adolescent spine fusion surgery. Spine (Phila Pa 1976) 24:219-224, 1999

10. Daubs MD, Lenke LG, Cheh G, Stobbs G, Bridwell KH: Adult spinal deformity surgery: complications and outcomes in patients over age 60. Spine (Phila Pa 1976) 32:22382244, 2007

11. Diebo BG, Passias PG, Marascalchi BJ, Jalai CM, Worley NJ, Errico TJ, et al: Primary versus revision surgery in the setting of adult spinal deformity: a nationwide study on 10,912 patients. Spine (Phila Pa 1976) 40:1674-1680, 2015

12. Drazin D, Shirzadi A, Rosner J, Eboli P, Safee M, Baron EM, et al: Complications and outcomes after spinal deformity surgery in the elderly: review of the existing literature and future directions. Neurosurg Focus 31(4):E3, 2011

13. Hu SS: Blood loss in adult spinal surgery. Eur Spine J $\mathbf{1 3}$ (Suppl 1):S3-S5, 2004

14. Kang JS, Moon KH, Kim BS, Yang SJ: Topical administration of tranexamic acid in hip arthroplasty. Int Orthop 41:259-263, 2017

15. Ker K, Edwards P, Perel P, Shakur H, Roberts I: Effect of tranexamic acid on surgical bleeding: systematic review and cumulative meta-analysis. BMJ 344:e3054, 2012

16. Kothari P, Somani S, Lee NJ, Guzman JZ, Leven DM, Skovr$\mathrm{lj}$ B, et al: Thirty-day morbidity associated with pelvic fixation in adult patients undergoing fusion for spinal deformity: a propensity-matched analysis. Global Spine J 7:39-46, 2017

17. Kratzer S, Irl H, Mattusch C, Bürge M, Kurz J, Kochs E, et al: Tranexamic acid impairs $\gamma$-aminobutyric acid receptor type A-mediated synaptic transmission in the murine amygdala: a potential mechanism for drug-induced seizures? Anesthesiology 120:639-649, 2014

18. Lin JD, Lenke LG, Shillingford JN, Laratta JL, Tan LA, Fischer CR, et al: Safety of a high-dose tranexamic acid protocol in complex adult spinal deformity: analysis of 100 consecutive cases. Spine Deform 6:189-194, 2018

19. Manji RA, Grocott HP, Leake J, Ariano RE, Manji JS, Menkis $\mathrm{AH}$, et al: Seizures following cardiac surgery: the impact of tranexamic acid and other risk factors. Can J Anaesth 59:6-13, 2012

20. McCall RE, Hayes B: Long-term outcome in neuromuscular scoliosis fused only to lumbar 5. Spine (Phila Pa 1976) 30:2056-2060, 2005

21. Murray DJ, Forbes RB, Titone MB, Weinstein SL: Transfusion management in pediatric and adolescent scoliosis surgery. Efficacy of autologous blood. Spine (Phila Pa 1976) 22:2735-2740, 1997
22. Nadler SB, Hidalgo JH, Bloch T: Prediction of blood volume in normal human adults. Surgery 51:224-232, 1962

23. Passias PG, Jalai CM, Worley N, Vira S, Marascalchi B, McClelland S III, et al: Adult spinal deformity: national trends in the presentation, treatment, and perioperative outcomes from 2003 to 2010. Spine Deform 5:342-350, 2017

24. Peters A, Verma K, Slobodyanyuk K, Cheriyan T, Hoelscher C, Schwab F, et al: Antifibrinolytics reduce blood loss in adult spinal deformity surgery: a prospective, randomized controlled trial. Spine (Phila Pa 1976) 40:E443-E449, 2015

25. Ramo BA, Roberts DW, Tuason D, McClung A, Paraison LE, Moore HG IV, et al: Surgical site infections after posterior spinal fusion for neuromuscular scoliosis: a thirty-year experience at a single institution. J Bone Joint Surg Am 96:2038-2048, 2014

26. Roberts I, Shakur H, Coats T, Hunt B, Balogun E, Barnetson L, et al: The CRASH-2 trial: a randomised controlled trial and economic evaluation of the effects of tranexamic acid on death, vascular occlusive events and transfusion requirement in bleeding trauma patients. Health Technol Assess 17:1-79, 2013

27. Sengupta DK, Mehdian SH, McConnell JR, Eisenstein SM, Webb JK: Pelvic or lumbar fixation for the surgical management of scoliosis in Duchenne muscular dystrophy. Spine (Phila Pa 1976) 27:2072-2079, 2002

28. Soroceanu A, Oren JH, Smith JS, Hostin R, Shaffrey CI, Mundis GM, et al: Effect of antifibrinolytic therapy on complications, thromboembolic events, blood product utilization, and fusion in adult spinal deformity surgery. Spine (Phila Pa 1976) 41:E879-E886, 2016

29. Thompson GH, Florentino-Pineda I, Poe-Kochert C: The role of Amicar in decreasing perioperative blood loss in idiopathic scoliosis. Spine (Phila Pa 1976) 30 (17 Suppl):S94-S99, 2005

30. White SJW, Cheung ZB, Ye I, Phan K, Xu J, Dowdell J, et al: Risk factors for perioperative blood transfusions in adult spinal deformity surgery. World Neurosurg 115:e731-e737, 2018

31. Winter SF, Santaguida C, Wong J, Fehlings MG: Systemic and topical use of tranexamic acid in spinal surgery: a systematic review. Global Spine J 6:284-295, 2016

32. Xie J, Lenke LG, Li T, Si Y, Zhao Z, Wang Y, et al: Preliminary investigation of high-dose tranexamic acid for controlling intraoperative blood loss in patients undergoing spine correction surgery. Spine J 15:647-654, 2015

33. Yu CC, Kadri O, Kadado A, Buraimoh M, Pawloski J, Bartol $\mathrm{S}$, et al: Intravenous and oral tranexamic acid are equivalent at reducing blood loss in thoracolumbar spinal fusion: a prospective randomized trial. Spine (Phila Pa 1976) 44:755761,2019

34. Yuan L, Zeng Y, Chen ZQ, Zhang XL, Mai S, Song P, et al: Efficacy and safety of antifibrinolytic agents in spinal surgery: a network meta-analysis. Chin Med J (Engl) 132:577588,2019

35. Zhang Y, Liu H, He F, Chen A, Yang H, Pi B: Does tranexamic acid improve bleeding, transfusion, and hemoglobin level in patients undergoing multilevel spine surgery? A systematic review and meta-analysis. World Neurosurg 127:289-301, 2019

36. Zheng F, Cammisa FP Jr, Sandhu HS, Girardi FP, Khan SN: Factors predicting hospital stay, operative time, blood loss, and transfusion in patients undergoing revision posterior lumbar spine decompression, fusion, and segmental instrumentation. Spine (Phila Pa 1976) 27:818-824, 2002

\section{Disclosures}

Dr. McDonagh: support of non-study-related clinical or research effort from Lungpacer Medical Inc. 


\section{Author Contributions}

Conception and design: Pernik, McDonagh, Bagley. Acquisition of data: Pernik, Dosselman, Walker, Hall, Peinado Reyes. Analysis and interpretation of data: Aoun, Pernik, Dosselman, Walker, Hall, Peinado Reyes. Drafting the article: Aoun, Pernik, Dosselman. Critically revising the article: Aoun, McDonagh, Bagley. Reviewed submitted version of manuscript: Aoun, McDonagh,
Bagley. Statistical analysis: Pernik, Dosselman. Study supervision: Bagley.

\section{Correspondence}

Salah G. Aoun: The University of Texas Southwestern, Dallas, TX.salah.aoun@phhs.org; salahaoun@hotmail.com. 